\title{
Analytical formulation of the elastic-plastic behaviour of bi-symmetrical steel shapes
}

\author{
António Manuel Baptista ${ }^{\mathrm{a} *}$, Jean-Pierre Muzeau ${ }^{\mathrm{b}}$ \\ ${ }^{a}$ Dep. Estruturas, LNEC, av. do Brasil 101, 1700-066 Lisboa Codex, Portugal \\ ${ }^{b}$ LGC, CUST, Blaise Pascal University, BP 206, 63174 Aubière Cedex, France
}

\begin{abstract}
This paper presents the analytical relationships of a non-linear model for the in-plane elasticplastic analysis of bi-symmetrical steel shapes bent about one of their main axes. The basic variables are the cross-section global deformations from which it is possible to evaluate the internal loads and the cross-section stiffness matrix components by means of simple expressions. Furthermore, the values of stresses and strains at any point of the cross-section may be determined knowing the values of the internal loads, in the elastic and elastic-plastic domains. The effects of progressive yielding spreading, as well as those of material strainhardening, are taken into account in the evaluation of the cross-section resistance capacity in the elastic-plastic domain. This analytical model represents an efficient, simple and accurate alternative to the elastic-plastic models based on numerical methods.
\end{abstract}

Keywords: Analytical model; Elastic-plastic behaviour; Cross-section; Global deformation interaction; Internal forces interaction; Strain-hardening; Resistance capacity; Deformation capacity; Stiffness

* Corresponding author: $\quad$ Tel.: +35121844 3252; fax: +35121 844 3025; E-mail address: ambaptista@lnec.pt 